\title{
2-year ADT improves survival in patients receiving high-dose radiotherapy
}

Men with intermediate-risk and high-risk prostate cancer treated with high-dose radiotherapy have better 5 -year overall and biochemical disease-free survival if they receive long-term androgen deprivation therapy (ADT) compared with short-term ADT, according to new data from the DART01/05 GICOR study.

In patients with high-risk disease, the benefits of combination treatment using high-dose radiotherapy and ADT have been demonstrated; however, how long ADT should be given to patients in this setting remained unclear. Almudena Zapatero and colleagues from Spain now report the results from their open-label, randomized, controlled phase III trial, which investigated this question in men with localized T1c-T3b intermediate-risk or high-risk prostate adenocarcinoma.

All 355 randomized patients were treated with neoadjuvant ADT (goserelin and flutamide or bicalutamide) for 2 months before receiving $3 \mathrm{D}$ conformal radiotherapy (median total dose $78 \mathrm{~Gy}$ ) with concomitant goserelin for a further 2 months (total ADT duration 4 months). 177 patients (50\%) who had been randomized to the long-term ADT arm of the study continued to receive goserelin for another 2 years. The primary study end point was biochemical diseasefree survival (failure was defined as a PSA concentration increase $\geq 2 \mathrm{ng} / \mathrm{ml}$ above nadir) and secondary end points included overall and metastasis-free survival.

\section{4 ...the benefit of long-term}

ADT was more evident in men with high-risk disease... 77

After a median follow-up period of 63 months, in the long-term and shortterm ADT arms, 5-year biochemical disease-free survival was $90 \%$ and $81 \%$ (HR 1.88), overall survival was $95 \%$ and
86\% (HR 2.48) and metastasis-free survival was $94 \%$ and $83 \%$ (HR 2.31), respectively. For all three outcomes, the benefit of long-term ADT was more evident in men with high risk disease than in men with intermediate-risk disease. Late rectal and urinary toxicity was similar in both groups.

"Our next goal is to determine the effect of long-term ADT in patients with unfavourable intermediate-risk prostate cancer and to investigate the relationship between testosterone recovery and outcome," says Almudena. "We will also concentrate our work on the analysis of potential cardiovascular morbidity according to prior patient comorbidities."

\section{Clemens Thoma}

Original article Zapatero, A. et al. High-dose radiotherapy
with short-term or long-term androgen deprivation
in localised prostate cancer (DART01/05 GICOR):
a randomised, controlled, phase 3 trial. Lancet Oncol.
doi:10.1016/S1470-2045(15)70045-8

